\title{
Control of Luminescence Properties in Naphthalene Diimide-Based Gel with Azodibenzoic Acid by Charge Transfer Interaction
}

\author{
Ji Ha Lee, Jaehyeon Park, and Jong Hwa Jung* \\ Department of Chemistry and Research Institute of Natural Sciences, Gyeongsang National University, \\ Jinju 660-701, Korea. *E-mail: jonghwa@gnu.ac.kr \\ Received May 2, 2014, Accepted May 16, 2014
}

Key Words : Diimide, Charge transfer, Life time, Rheology

In recent years, the self-assembly of various organic $\pi$ systems, mediated by non-covalent bonding, have been topics of considerable interest. ${ }^{1}$ These compounds that can undergo gelation hold great promise as building blocks for materials that can be applied in the construction of optoelectronic devices. ${ }^{2,3}$ The self-sorting between aromatic compounds that can serve as electron donors and electron acceptors allows the formation of distinct p- and n-type bundles of fibers that further intermix and generate multiple junction points for efficient electron transfer and transport of the charge carriers. However, a difficulty associated with simple mixing of such compounds is the possibility of a different mode of assembly, i.e., charge-transfer interaction mediated by alternate donor-acceptor stacking. Thus, examples related to self-sorting among donor-acceptor chromophores have been limited. ${ }^{4}$ Recently, Ghosh and colleagues has demonstrated a hydrogen-bonding mediated self-assembly, ${ }^{5-9}$ in a mixture of bis-amide-functionalized naphthalene-diimide and dialkoxy-naphthalene, where mutual recognition between similar chromophores took place to maximize the effect of hydrogen-bonding.

Naphthalene-diimide derivatives have been extensively explored as building blocks for the generation of various supramolecularly assembled systems in organic- or aqueous media, because of their n-type semiconductivity, propensity for $\pi$-stacking and electron-accepting nature. ${ }^{9-12}$ Examples of such systems include organogels, catenanes, rotaxanes, foldamers, nano-tubes, hydrogels, nanoparticles, supramolecular photosystems and synthetic ion channels. ${ }^{13-16}$

To date, the literature contains little information regarding the photoluminescence properties of hydrogels, including switching of the emission color, field emission and light emitting properties. ${ }^{5,9}$ In view of the high photoluminescence quantum efficiencies of naphthalene diimide derivatives used as fluorescent dyes, ${ }^{5,17}$ we expected such compounds to exhibit interesting photoluminescence properties by charge transfer interaction with guest molecules. Herein, we report the formation of a hydrogel of naphthalene-diimide (1) with 4,4'-azodibenzoic acid (2). The luminescence properties were controlled by the addition of $\mathbf{2}$. Furthermore, the life-times of hydrogels were enhanced by the charge-transfer interactions.

The gel based on 1 was prepared by dissolving $1 \%$ (by
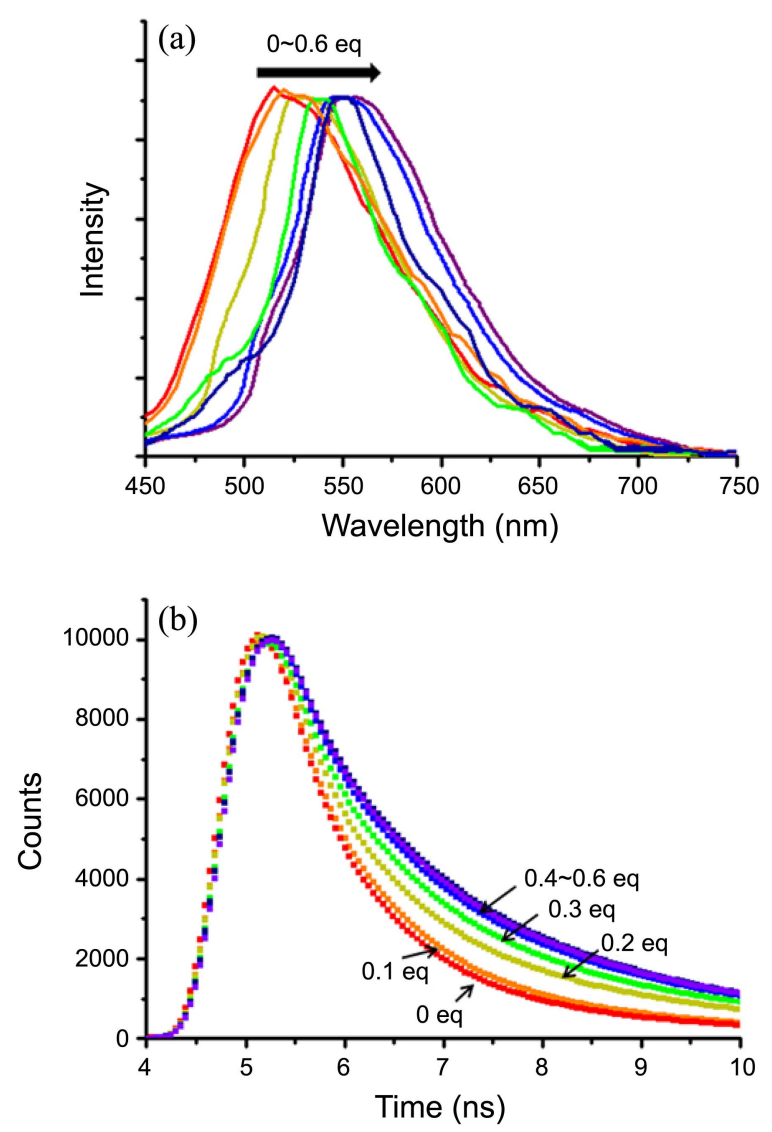

Figure 1. (a) Fluorescence spectra and (b) lifetime decay profiles of hydrogel 1 with different concentration of 2 (0-0.6 equivalent).

weight) of $\mathbf{1}$ in water. To this solution a small volume of $\mathbf{2}$ as an additive in water was added in concentrations varying from 0.2-1.0 equivalents with respect to the gelator 1 concentration. The samples were then left to stand for a week. As shown in Fig. S1, 1 could be gelated in the presence of 0 0.6 equivalent of $\mathbf{2}$, but $\mathbf{1}$ could not gelate when the amount of added 2 exceeded 0.7 equivalent. These gels prepared from a mixture of $\mathbf{1}$ and $\mathbf{2}$ exhibited a strong emission with a variety of colors under UV irradiation, in which the emission colors were dependent on the concentration of 2 .

The luminescent spectra of hydrogel 1 with varying concentrations of $\mathbf{2}$ were observed by fluorimetry (Fig. 1(a)). 

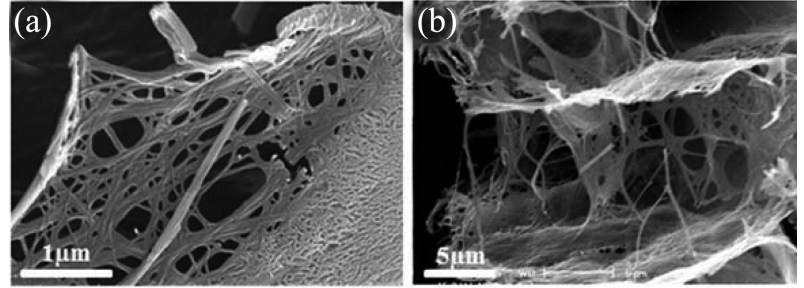

Figure 2. SEM images of hydrogel $\mathbf{1}$ (a) without and (b) with 0.6 equivalent of 2 .

The luminescence spectrum of gel $\mathbf{1}$ in the absence of $\mathbf{2}$ showed an absorption band with a maximum at $520 \mathrm{~nm}$, which appears as a blue color. In contrast, upon the addition of aliquots of 2, the luminescence spectra of gel $\mathbf{1}$ were gradually shifted to longer wavelengths. These red shifts to the charge transfer interaction between $\mathbf{1}$ and $\mathbf{2}$, with gelator $\mathbf{1}$ acting as the electron acceptor whereas $\mathbf{2}$ acts as the electron donor.

In order to understand the excited-state behavior of the hydrogel 1 with 2, we conducted a time-resolved emission study of hydrogel 1 with different concentrations of $\mathbf{2}$ (Fig. 1B). In the sol state, the luminescence lifetime was found to be $1.82 \mathrm{~ns}$ (Table 1). The short lifetime of $\mathbf{1}$ alone was due to 1 itself in the sol state.

At $1.0 \mathrm{wt} \%$ of $\mathbf{1}$ in the absence of $\mathbf{2}$ in the gel state, the lifetime, termed the $\tau$ value, was longer than that observed with $0.5 \mathrm{wt} \%$ of $\mathbf{1}$ in the sol state. The addition of $\mathbf{2}$ to hydrogel $\mathbf{1}$ induced a slightly longer lifetime as compared to the hydrogel 1 without 2 . An increase in the concentration of 2 to hydrogel 1 led to further increases of life-time $(19.3 \mathrm{~ns})$ value. In the presence of $\mathbf{2}$, the charge-transfer interaction between 1 and 2 was relatively stronger than that of 1 by itself. Therefore, the emission lifetime of hydrogel $\mathbf{1}$ could be controlled by increasing the concentration of $\mathbf{2}$.

The morphology of hydrogel 1 without and with 2 (0.2-0.6 equivalent) prepared from water was observed by scanning electron microscopy (SEM). The morphology of hydrogel 1 alone exhibited a fiber structure of 50-60 $\mathrm{nm}$ in diameter and several $\mu \mathrm{m}$ in length. Based on SEM images, increasing the concentration of $\mathbf{2}$ under conditions that successfully formed hydrogel $\mathbf{1}$ had a negligible effect, producing a similar nanofiber structure with diameters of 50-60 nm (Fig. 2).

NMR is a powerful technique for studying gels on the

Table 1. Life-times of Hydrogel 1 with Different Concentration of 2

\begin{tabular}{cccc}
\hline Concentration of $\mathbf{1}$ & Equivalent of $\mathbf{2}$ & Lifetime $(\tau)$ & State \\
\hline $0.5 \mathrm{wt} \%$ & 0 equivalent & $1.8 \mathrm{~ns}$ & Sol \\
$1.0 \mathrm{wt} \%$ & 0 equivalent & $9.1 \mathrm{~ns}$ & Gel \\
$1.0 \mathrm{wt} \%$ & 0.1 equivalent & $10.8 \mathrm{~ns}$ & Gel \\
$1.0 \mathrm{wt} \%$ & 0.2 equivalent & $12.8 \mathrm{~ns}$ & Gel \\
$1.0 \mathrm{wt} \%$ & 0.3 equivalent & $14.7 \mathrm{~ns}$ & Gel \\
$1.0 \mathrm{wt} \%$ & 0.4 equivalent & $16.2 \mathrm{~ns}$ & Gel \\
$1.0 \mathrm{wt} \%$ & 0.5 equivalent & $17.9 \mathrm{~ns}$ & Gel \\
$1.0 \mathrm{wt} \%$ & 0.6 equivalent & $19.3 \mathrm{~ns}$ & Gel \\
\hline
\end{tabular}

molecular scale, as it provides a way to quantify what is present in the 'liquid-like' phase, and what is immobilized in the 'solid-like' gel fibers, as documented previously by Escuder et al. and Smith group. ${ }^{4,18,19}$ NMR peaks were observed for mobile components, whereas anything immobilized, for example within the gel fibers, would be broadened into the baseline. We used variable temperature (VT) NMR to examine the role of heat in the response of $\mathbf{2}$ in the presence of gelator 1. We measured the NMR spectrum 2 (1.3 mg, 0.4 equiv.) in the presence of $1(5.0 \mathrm{mg})$ in $\mathrm{D}_{2} \mathrm{O}(0.5 \mathrm{~mL})$ at increasing temperatures. By integrating an NMR peak associated with $\mathbf{1}$ and $\mathbf{2}$, and referencing the integral to that associated with the mobile $\mathrm{D}_{2} \mathrm{O}$ solvent molecules, we gained a quantitative insight into the relative amounts of mobile gelator $\mathbf{1}$ and $\mathbf{2}$. In this analysis, we assumed that the contribution from immobile gelator $\mathbf{1}$ and $\mathbf{2}$ would be zero. As the temperature decrease, the ratio of solvent: $\mathbf{1}$ and $\mathbf{2}$ increased. The gelator $\mathbf{1}$ and $\mathbf{2}$ became immobilized as the temperature decreased and the sample was transformed into a gel. The largest changes in peak height and, by inference, gelator mobility, occurred around $40-70{ }^{\circ} \mathrm{C}$ (Fig. S2). Furthermore, these results demonstrated that molecules of $\mathbf{2}$ were incorporated into gel $\mathbf{1}$ in the molecular level in terms of establishing molecular recognition pathways.

Rheological data provides information about the behavior of hydrogels when they are exposed to mechanical stress, especially the "storage" (or "elastic") modulus G', which represents the ability of the deformed material to "snap back" to its original geometry, and the "loss" (or "viscous") modulus G", which represents the tendency of a material to flow under stress. Two rheological criteria required for a metallogel are: (i) the independence of the dynamic elastic modulus, $\mathrm{G}^{\prime}$, with respect to the oscillatory frequency, and (ii) $\mathrm{G}^{\prime}$ must exceed the loss modulus $\mathrm{G}^{\prime \prime}$ by about 1 order of magnitude.

We first used dynamic strain sweep to determine the proper conditions for the dynamic frequency sweep of the hydrogel at different concentrations of 2 . As shown in Fig. $\mathrm{S} 3$, the values of the storage modulus $\left(\mathrm{G}^{\prime}\right)$ and the loss modulus $\left(\mathrm{G}^{\prime \prime}\right)$ exhibited a weak dependence from 0.1 to $1.0 \%$ of strain (with $\mathrm{G}^{\prime}$ dominating $\mathrm{G}^{\prime \prime}$ ), indicating that the sample is a hydrogel. The values of both $\mathrm{G}^{\prime}$ and $\mathrm{G}^{\prime \prime}$ of the hydrogel in the presence of 0.6 equivalents of $\mathbf{2}$ were remarkably enhanced in comparison to the hydrogels with only 0.2 or 0.4 equivalents of 2 . These results reflect the stabilization of the hydrogel by charge-transfer interactions in the presence of 0.6 equivalent of 2 .

We used dynamic frequency sweep to study the hydrogel after setting the strain amplitude at $0.01 \%$ (within the linear response region of the strain amplitude). $G^{\prime}$ and $G^{\prime \prime}$ were almost constant with the increase of frequency from 0.1 to $100 \mathrm{rad} / \mathrm{s}$ (Fig. S4). The value of $\mathrm{G}^{\prime}$ was about 2-5 times larger than that of $\mathrm{G}^{\prime \prime}$ over the whole range (0.1-100 $\left.\mathrm{rad} / \mathrm{s}\right)$, suggesting that the hydrogel was fairly tolerant to external force. As observed by changes of dynamic strain sweep, the values of both $\mathrm{G}^{\prime}$ and $\mathrm{G}^{\prime \prime}$ of the hydrogel at values above 0.6 equivalent of 2 concentration were 10 time larger than that 
of the hydrogel in the presence of 0.2 equivalent of 2 . All values of $\mathrm{G}^{\prime}$ and $\mathrm{G}^{\prime \prime}$ in the hydrogel were strongly influenced by the concentration of $\mathbf{2}$ with respect to 1 .

In conclusion, we have controlled the luminescent properties of hydrogel 1 upon addition of azodibenzoic acid through charge-transfer interactions. These hydrogels showed nanosecond-scale emission decay, in which emission lifetimes were dependent on the concentration of the azodibenzoic acid $\mathbf{2}$. The azodibenzoic acid was incorporated into hydrogel 1 at the molecular level. Together, measurements of dynamic oscillation and steady shear indicated the formation of a strong and thermally stable network whose supramolecular structure was reversible by mechanical and thermal treatments.

\section{Experimental}

Materials and Methods. ${ }^{1} \mathrm{H}$ and ${ }^{13} \mathrm{C}$ NMR spectra were measured on a Bruker ARX 300 apparatus. IR spectra were obtained for $\mathrm{KBr}$ pellets, in the range $400-4000 \mathrm{~cm}^{-1}$, with a Shimadzu FT-IR 8400S, and Mass spectra were obtained by a JEOL JMS-700 mass spectrometer. The optical absorption spectra of the samples were obtained at $298 \mathrm{~K}$ using a UVvis spectrophotometer (Hitachi U-2900). All fluorescence spectra were recorded in RF-5301PC spectrophotometer.

Fluorescence Lifetime Measurements. Emission lifetime measurements were performed using a conventional laser system. The excitation source used was the $420 \mathrm{~nm}$ output of a Spectra-Physics Quanta-Ray Q-switched GCR-150-10 pulsed Nd:YAG lager. Luminescence decay signals were detected by Hamamatsu R928 PMT, recorded on a Tektronix model TDS-620A (500 MHz, $2 \mathrm{GS} / \mathrm{s}$ ) digital oscilloscope and analyzed using a program for exponential fits.

Rheological Measurements. These were carried out on freshly prepared gels using a controlled stress rheometer (AR-1000N, TA Instruments Ltd., New Castle, DE, USA). Parallel plate geometry of $40 \mathrm{~mm}$ diameter and $1.5 \mathrm{~mm}$ gap was employed throughout. Following loading, the exposed edges of samples were covered with a silicone fluid from BDH (100 cs) to prevent water loss. Dynamic oscillatory work kept a frequency of $1.0 \mathrm{rad} \mathrm{s}^{-1}$. The following tests were performed: increasing amplitude of oscillation up to $100 \%$ apparent strain on shear, time and frequency sweeps at $25^{\circ} \mathrm{C}$ (60 min and from $0.1-100 \mathrm{rad} \mathrm{s}^{-1}$, respectively). Unidirectional shear routines were performed at $25^{\circ} \mathrm{C}$ covering a shear-rate regime between $10^{-1}$ and $10^{3} \mathrm{~s}^{-1}$. Mechanical spectroscopy routines were completed with transient measurements. In doing so, the desired stress was applied instantaneously to the sample and the angular displacement was monitored for $60 \mathrm{~min}$ (retardation curve). After completion of the run, the imposed stress was withdrawn and the extent of structure recovery was recorded for another $60 \mathrm{~min}$ (relaxation curve). Dynamic and steady shear measurements were conducted in triplicate and creep (transient) measurements in duplicate.

SEM Observation. Scanning electron micrographs of the samples were taken with a field emission scanning electron microscope (FE-SEM, Philips XL30 S FEG). The accelerating voltage of SEM was $5-15 \mathrm{kV}$ and the emission current was $10 \mu \mathrm{A}$.

${ }^{1}$ H NMR Spectroscopy. The hydrogel $(\mathbf{1}+\mathbf{2})$ was made in $\mathrm{D}_{2} \mathrm{O}$ at 5.0 and $1.3 \mathrm{mg}$, respectively. For the temperature variable experiment, sample solutions were heated from 25 ${ }^{\circ} \mathrm{C}$ to higher temperature with an external temperature controller and the spectral measurements were carried out at different temperatures. On reaching the desired temperature, 10 min equilibrium time was provided before each measurement. The NMR experiment was performed in 300 and 500 $\mathrm{MHz}$ spectrometers. For each reading, 100 scans were taken with 1 sec delay time.

Preparation of Binary Gels with 2. In a vial, compound $1(5 \mathrm{mg})$ was dissolved in water $(200 \mu \mathrm{L})$. Compound $2(0-$ 0.8 equivalent) was added to the soltion $\mathbf{1}$, then was heated until solution. The solution was maintained at room temperature, and was formed the gel in ambient temperature.

Compound 1. A mixture of 1,4,5,8-naphthalene-tetracarboxylic dianhydride (NDA) $(0.8 \mathrm{~g}, 3 \mathrm{mmol})$ and 4aminopyridine $(6 \mathrm{mmol})$ in DMF $(20 \mathrm{~mL})$ was heated under reflux for $8 \mathrm{~h}$. When the reaction mixture reached room temperature, a crystalline solid precipitated out, which was collected by filtration. The crude product was purified by recrystallization from DMF to obtain $1 .{ }^{1} \mathrm{H}$ NMR $(300 \mathrm{MHz}$, DMSO- $\left.d_{6}\right) \delta 8.81\left(\mathrm{dd}, J=1.6 \mathrm{~Hz}\right.$ and $\left.3 \mathrm{~Hz}, 4 \mathrm{H}_{\mathrm{c}}\right), 8.75(\mathrm{~s}$, $\left.4 \mathrm{H}_{\mathrm{a}}\right)$, and $7.58\left(\mathrm{dd}, J=1.6 \mathrm{~Hz}\right.$ and $\left.4.5 \mathrm{~Hz}, 4 \mathrm{H}_{\mathrm{b}}\right) \mathrm{ppm} .{ }^{13} \mathrm{C}$ NMR (75 MHz, DMSO- $\left.d_{6}\right) \delta 162.80,151.23,144.01$, 131.01, 127.43, 127.21 and 124.96 ppm. MS (HR-ESI, +ve) $m / z$ : Observed $421.0942[\mathrm{M}+\mathrm{H}]^{+},[\mathrm{M}+\mathrm{H}]_{\text {calcd }}^{+}=421.0937$. FT IR: $3069.97,1712.27,1661.71,1574.39,1491.67 \mathrm{~cm}^{-1}$.

Compound 2. Compound 2 was purchased from Aldrich, and used without further purification.

Acknowledgments. This work was supported by a grant from NRF (2012R1A4A1027750 and 2012R1A2A2A01002547 and 2014M2B2A9030338). In addition, this work was partially supported by a grant from the Next-Generation BioGreen 21 Program (SSAC, grant\#: PJ009041022012), Rural development Administration, Korea.

Supporting Information. Gelation test, VT ${ }^{1} \mathrm{H}$ NMR experiment data and rheometer data.

\section{References}

1. Chen, Z.; Lohr, A.; Saha-Möller, C. R.; Würthner, F. Chem. Soc. Rev. 2009, 38, 564.

2. Hains, A. W.; Liang, Z.; Woodhouse, M. A.; Gregg, B. A. Chem. Rev. 2010, 110, 6689.

3. Babu, S. S.; Prasanthkumar, S.; Ajayaghosh, A. Angew. Chem. Int, Ed. 2012, 51, 1766

4. Smith, M. M.; Smith, D. K. Soft Matter 2011, 7, 4856.

5. Molla, M. R.; Gehrig, D.; Roy, L.; Kamm, V.; Paul, A.; Laquai F.; Ghosh, S. Chem. Eur. J. 2013, 20, 760 .

6. Das, A.; Molla, M. R.; Maity, B.; Koley, D.; Ghosh, S. Chem. Eur. J. 2012, 18, 9849 .

7. Molla, M. R.; Ghosh, S. Chem. Eur. J. 2012, 18, 9860.

8. Kar, H. K.; Molla, M. R.; Ghosh, S. Chem. Commun. 2013, 4220. 
9. Das A.; Ghosh, S. Angew. Chem. Int, Ed. 2014, 126, 1110.

10. Bhosale, S. V.; Jani, C. H.; Langford, S. Chem. Soc. Rev. 2008, 37 , 331.

11. Bjosale, R.; Míšek, J.; Sakai, N.; Matile, S. Chem. Soc. Rev. 2010, 39, 138.

12. Dawson, R. E.; Henning, A.; Weimann, D. P.; Emery, D.; Ravikumar, V.; Montenegro, J.; Takeuchi, T.; Gabutti, S.; Nayor, N.; Mareda, J.; Schalley, C. A.; Matile, S. Nat. Chem. 2010, 2, 533.

13. Talukdar, P.; Bollot, G.; Mareda, J.; Sakai, N.; Matile, S. J. Am. Chem. Soc. 2005, 127, 6528.
14. Vignon, S. A.; Jarrosson, T.; Iijima, T.; Tseng, H.-R.; Sanders, J. K. M.; Stoddart, J. F. J. Am. Chem. Soc. 2004, 126, 9884.

15. Au-Yeung, H. Y.; Pantos, G. D.; Sanderrs, J. K. M. Proc. Natl. Acad. Sci. USA 2009, 106, 10466.

16. Bhosale, S. V.; Jani, C. H.; Langford, S. J. Chem. Soc. Rev. 2008, 37, 331 .

17. Kumar, M.; George, S. J. Nanoscale 2011, 3, 2130.

18. Edwards, W.; Smith, D. K. J. Am. Chem. Soc. 2013, 135, 5911.

19. Hirst, A. R.; Miranet, J. F.; Escuder, B.; Noirez, L.; Castelletto, V.; Hamley, I. W.; Smith, D. K. Chem. Eur. J. 2009, 15, 372. 